\title{
A comparison of quality and cost indicators by surgical specialty for lobectomy of the lung
}

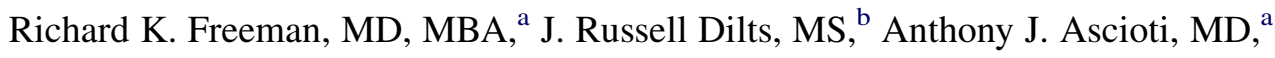 \\ Theresa Giannini, ARNP, ${ }^{a}$ and Raja J. Mahidhara, MD $^{\mathrm{a}}$
}

\begin{abstract}
Objectives: This investigation compared patients undergoing lobectomy for non-small cell lung cancer by either a general surgeon or a cardiothoracic surgeon across a geographically diverse system of hospitals to see whether a significant difference in quality or cost was present.
\end{abstract}

\begin{abstract}
Methods: The Premiere administrative database and tumor registry data of a single health system's hospitals was used to compare adherence to national treatment guidelines, patient outcomes, and charges for patients undergoing lobectomy for non-small cell lung cancer in a 5-year period. Surgeons performing lobectomy were designated as a general surgeon or cardiothoracic surgeon according to their national provider number and board certification status. Excluded from analysis were centers that performed fewer than 50 lobectomies during the study period.
\end{abstract}

Results: During the study period, 2823 lobectomies were performed by 46 general surgeons and 3653 lobectomies were performed by 29 cardiothoracic surgeons in 54 hospitals in a single health care system. Significant differences were found between general and cardiothoracic surgeons with respect to adherence to national guidelines in staging and treatment, mean length of stay, significant morbidity, and operative mortality. Mean charges for lobectomy of the lung were also found to differ significantly between general and cardiothoracic surgeons.

Conclusions: This review found that currently measurable indicators for quality of care were significantly superior and overall charges were significantly reduced when a lobectomy for non-small cell lung cancer was performed by a cardiothoracic surgeon rather than by a general surgeon. (J Thorac Cardiovasc Surg 2013;145:68-74)

According to some estimates, the majority of lung resections in the United States continue to be performed by general surgeons. ${ }^{1}$ Other surgical specialties have found that surgeons with additional formal training after general surgery residency achieve superior outcomes in more complex surgical procedures relative to general surgeons. ${ }^{2,3}$ Several investigations have assessed the issue of whether a similar variation in results exists between residency-trained cardiothoracic surgeons and general surgeons. ${ }^{1,4-6}$ Although these authors have been able to show variation in such things as preoperative and intraoperative staging, long-term survival, and operative mortality, these analyses are limited because of the use of a combination of claims or nonconsecutive sampling databases, case mix designation of surgeon specialty rather than board certification, or the inclusion of a mixture of surgical procedures for analysis. Furthermore,

From the Department of Thoracic and Cardiovascular Surgery, ${ }^{a}$ St Vincent Hospital, Indianapolis, Ind; and Ascension Health, ${ }^{\mathrm{b}}$ St Louis, Mo.

Disclosures: Authors have nothing to disclose with regard to commercial support.

Read at the 92nd Annual Meeting of The American Association for Thoracic Surgery, San Francisco, California, April 28-May 2, 2012.

Received for publication April 8, 2012; revisions received Aug 14, 2012; accepted for publication Sept 12, 2012; available ahead of print Oct 10, 2012.

Address for reprints: Richard K. Freeman, MD, 8433 Harcourt Rd, Indianapolis, IN 46260 (E-mail: RFreeman@corvascmds.com).

0022-5223/\$36.00

Copyright (c) 2013 by The American Association for Thoracic Surgery

http://dx.doi.org/10.1016/j.jtcvs.2012.09.012 no attempt at comparing the costs of care between the two surgical specialties has been made. The purpose of this investigation was to compare the adherence to nationally accepted treatment guidelines, outcomes, and associated costs for lobectomy of the lung for non-small cell lung cancer (NSCLC) between general surgeons and thoracic surgeons within a diverse health care system.

\section{MATERIALS AND METHODS}

This study was designed as a retrospective cohort analysis. Institutional review board approval was obtained at St Vincent Hospital (Indianapolis, Ind) and individual patient consent was not required with the condition of patient anonymity outside the initial data-gathering phase of the study. By interrogating the Premier inpatient database (Premier Inc, Charlotte, NC), hospitals performing at least 50 lobectomies (cumulative; Current Procedural Terminology [CPT] code 32480) for NSCLC (International Classification of Diseases, Ninth Revision [ICD-9] diagnosis codes $162.2,162.3,162.4,162.5,162)$ during the calendar years 2005 through 2009 within the Ascension Health system were identified. Lobectomy procedures were then attributed to individual surgeons within these facilities. Surgeons were identified as either a general surgeon or a thoracic surgeon on the basis their national provider numbers and their board certification status. No attempt was made to differentiate thoracic surgeons who practiced cardiac and thoracic surgery from those that limited their practice to noncardiac thoracic surgery.

Patient demographic data; adherence to National Comprehensive Cancer Network (NCCN) guideline ${ }^{7}$; and information related to the lobectomy procedure, including charges, postoperative stay, operative morbidities, and operative mortality were abstracted for each patient from the Premier database or the local hospital's American College of Surgeons tumor 


\section{Abbreviations and Acronyms \\ $\mathrm{NCCN}=$ National Comprehensive Cancer Network \\ NSCLC $=$ non-small cell lung cancer}

registry data (Table 1). Also calculated were Charlson comorbidity index scores for each patient, as determined from secondary ICD-9 disease codes. ${ }^{8,9}$ Excluded from analysis were patients undergoing a bilobectomy (CPT 32482), lobectomy for a superior sulcus tumor or pulmonary metastasis (ICD-9 197), lobectomy with a bronchoplastic procedure (CPT 32486), video-assisted thoracoscopic lobectomy, or lobectomy in the setting of stage IV NSCLC, such as a patient with an isolated brain metastasis. Also excluded were patients undergoing lobectomy with a primary ICD-9 code denoting an endobronchial (162.9) or tracheal (162) tumor component, NSCLC involving multiple lobes of the lung (162.8) or the chest wall $(198.89,171.4)$, or a pleura-based neoplasm (163).

Postoperative stay was calculated as the number of days from the day of surgery until death or discharge. Patients who died after lobectomy did not have the opportunity for readmission and so were removed from that portion of the analysis. Postoperative morbidities were attributed in a binary fashion by patient, so that a patient who had more than 1 event was only counted once; however the tabulations of specific categories of complications include every occurrence. Operative mortality was defined as patient death after surgery before discharge from the hospital or within 30 days of surgery.

Hospital charges represent the total gross charges before any contractual adjustments and include all postings from the day of surgery through discharge or death. Professional fees were excluded. Parity of hospital charges was tested by examining each facility's charge per discharge index; a comparison of inpatient charges indexed to discharge diagnoses. ${ }^{10}$ This is a method of determining whether different facilities have similar levels of charges used by the private insurers and the Centers for Medicare and Medicaid Services. ${ }^{11}$ Patients transferred between facilities during their lobectomy hospitalization were excluded from eligibility from this study because of the artifactual change in charges and hospital stay that resulted. A readmission was attributed to patients designated in either cohort who required readmission to the hospital within 30 days of discharge from the lobectomy admission; however, charges incurred during the readmission were not included in the hospital charge calculation for that patient.

The unit of analysis for this study was a patient undergoing lobectomy for NSCLC. Surgeon specialty was identified as the exposure variable. Discharge versus operative mortality was the primary outcome for the analysis. Multiple logistic regression analysis was used to study relationships between patient variables and the identified outcome measures. Regression models were adjusted for clustering or nesting at the hospital level by the construction of 2-level hierarchic analysis models with the assumption of normative distribution of variables. ${ }^{11,12}$ Group mean centering for each cohort was used within the analyses. Adjustments within the regression analysis were made for patient age and Charlson comorbidity index score and reported as adjusted odds ratios. Hospital and surgeon volume were then individually adjusted by including a continuous variable representing each in the regression model and the effect on the primary outcomes observed.

Bivariate analysis of data was performed with GraphPad Prism software (version 4.02; GraphPad Software, Inc, San Diego, Calif) for Windows (Microsoft, Redmond Wash). Differences between categoric variables were evaluated by the Fisher's exact test. Differences between continuous variables were measured by the 2-tailed Student $t$ test or the Mann-Whitney test for nonnormally distributed data. Multivariate analysis was performed with Stata software (version 11; StataCorp, College Station, Tex).

For the purposes of this investigation, adherence to NCCN guidelines (Table 2) was defined according to whether pulmonary function testing, computed tomographic imaging of the chest, and staging of the mediastinum occurred before surgery. Mediastinal lymph node sampling or dissection at the time of lobectomy was also required.

\section{RESULTS}

During the 5-year study period, 2823 lobectomies that were performed by 46 general surgeons and 3653 lobectomies that were performed by 29 thoracic surgeons at 54 hospitals in 31 states met the entrance criteria for this investigation (Table 3). This resulted in mean numbers of lobectomies for general surgeons and thoracic surgeons of 61 and 126, respectively, during the 5-year period. Mean age and mean Charlson comorbidity index score varied significantly between the two patient cohorts. Sex distribution did not.

The frequencies of patients with pathologic stage I and II NSCLC were similar between the 2 patient groups; however, significantly more patients with stage IIIA disease underwent surgery by a thoracic surgeon, whereas significantly more patients with stage IIIB disease underwent surgery in the general surgeon cohort. It was also significantly more common for patients with stage III NSCLC to receive neoadjuvant therapy if their care involved a thoracic surgeon.

Patients operated on by a thoracic surgeon also had a significantly higher rate of fulfilling the study's NCCN criteria. This finding was true for each of the 4 criteria examined as well as for the overall category. Similarly significant was the difference in the number of patients found not to have a malignancy in the lobectomy specimen in the general surgeon group relative to the thoracic surgeon cohort.

Mean hospital stay was significantly longer in the general surgeon cohort than in the thoracic surgeon cohort. In addition, the number of patients with a prolonged stay, defined by Kozower and colleagues ${ }^{11}$ as longer than 14 days, was significantly greater in the general surgeon patient cohort. No significant difference in readmission rates between the 2 groups was found. The difference in stay in the general surgeon cohort could not be explained solely on the basis of the differences in operative morbidity and mortality.

The frequency of postoperative morbidities was found to be significantly higher in the general surgeon patient cohort than in the thoracic surgeon patient cohort (Table 4). When individual postoperative events were compared, prolonged air leak and return to the operating room for bleeding were found to differ significantly between the 2 patient groups, with a higher frequency of occurrence in the general surgeon patient cohort. Operative mortality was also significantly more frequent in the general surgeon patient cohort. No variables examined other than surgeon specialty were found to be predictors for morbidity or mortality after lobectomy for NSCLC.

After adjustment for the confounding variables of age and Charlson comorbidity index score, multiple logistic 
TABLE 1. Availability of data points from the Premier database and the local hospital's American College of Surgeons tumor registry

\begin{tabular}{lcc}
\hline \multicolumn{1}{c}{ Variable } & Premier & $\begin{array}{c}\text { Tumor } \\
\text { registry }\end{array}$ \\
\hline Age & $\mathrm{x}$ & $\mathrm{x}$ \\
Sex & $\mathrm{x}$ & $\mathrm{x}$ \\
Pulmonary function testing & $\mathrm{x}$ & \\
Computed tomographic imaging of the chest & $\mathrm{x}$ & \\
Prelobectomy mediastinal staging & $\mathrm{x}$ & $\mathrm{x}$ \\
Neoadjuvant chemotherapy or radiation therapy & $\mathrm{x}$ & $\mathrm{x}$ \\
Intraoperative mediastinal staging & $\mathrm{x}$ & $\mathrm{x}$ \\
Pathologic stage* & & $\mathrm{x}$ \\
Charlson comorbidity index & $\mathrm{x}$ & \\
No finding of malignancy after lobectomy & $\mathrm{x}$ & $\mathrm{x}$ \\
Readmission & $\mathrm{x}$ & \\
Morbidity & $\mathrm{x}$ & \\
Mortality & $\mathrm{x}$ & $\mathrm{x}$ \\
Charges & $\mathrm{x}$ & \\
*Staging according to Edge SB, Byrd DR, Compton CC, Fritz AG, Greene FL, Trotti \\
A, editors. AJCC cancer staging manual. 7th ed. New York: Springer; 2010.
\end{tabular}

regression analysis found the rate of operative mortality to be independently reduced by $63 \%$ (adjusted odds ratio, $0.37 ; 95 \%$ confidence interval $0.13-0.59 ; P<.0001$ ) for patients undergoing lobectomy by a thoracic surgeon when compared with the general surgeon patient cohort. An analysis adjusting for differences in both hospital and provider volume found a reduction in mortality; however, the significant difference remained between the 2 cohorts (adjusted odds ratio, $0.60 ; 95 \%$ confidence interval $0.53-0.72 ; P<.0001$ ).

Global parity among individual hospitals with respect to charges was found, with no significant difference recognized when charges per discharge indexes were compared; however, a significant difference between mean charges of $\$ 11,000(14 \%)$ was found between the general surgeon and thoracic surgeon cohorts $(P<.0001)$. An analysis of charge segregation found that $76 \%$ of the differences in mean charges between the groups were directly related to the length of stay and morbidities in the general surgeon cohort.

\section{DISCUSSION}

The concept that formal specialty training produces superior results for surgical procedures is not a new one. Several

TABLE 2. Scoring of National Comprehensive Cancer Network guideline $^{7}$ adherence

\begin{tabular}{lc}
\hline \multicolumn{1}{c}{ Variable } & \multicolumn{1}{c}{ Adherence } \\
\hline $\begin{array}{l}\text { Pulmonary function tests } \\
\text { before lobectomy }\end{array}$ & Results dated before lobectomy \\
$\begin{array}{c}\text { Computed tomographic } \\
\text { imaging before lobectomy }\end{array}$ & Results dated within $90 \mathrm{~d}$ before lobectomy \\
$\begin{array}{c}\text { Prelobectomy mediastinal } \\
\text { staging }\end{array}$ & $\begin{array}{c}\text { Positron emission tomography, } \\
\text { endobronchial ultrasonography, } \\
\end{array}$ \\
\hline
\end{tabular}

TABLE 3. Results of analysis

\begin{tabular}{|c|c|c|c|}
\hline & $\begin{array}{l}\text { General } \\
\text { surgeons }\end{array}$ & $\begin{array}{l}\text { Thoracic } \\
\text { surgeons }\end{array}$ & $\begin{array}{c}P \\
\text { value }\end{array}$ \\
\hline $\mathrm{N}$ & 2823 & 3653 & \\
\hline Age $(y$, mean $\pm S D)$ & $63 \pm 13$ & $67 \pm 17$ & $<.0001$ \\
\hline Male & $1502(53 \%)$ & $1932(53 \%)$ & .052 \\
\hline $\begin{array}{l}\text { Charlson comorbidity index } \\
\quad(\text { mean } \pm \mathrm{SD})\end{array}$ & $6 \pm 3$ & $9 \pm 4$ & $<.0001$ \\
\hline \multicolumn{4}{|l|}{ Pathologic stage } \\
\hline I & $1256(45 \%)$ & $1399(38 \%)$ & .21 \\
\hline II & $1077(38 \%)$ & $1569(43 \%)$ & .08 \\
\hline $\mathrm{III}_{\mathrm{A}}$ & $229(8 \%)$ & $547(15 \%)$ & $<.0001$ \\
\hline $\mathrm{III}_{\mathrm{B}}$ & $261(9 \%)$ & $138(4 \%)$ & $<.0001$ \\
\hline $\begin{array}{l}\text { National Comprehensive Cancer } \\
\text { Network guideline adherence }\end{array}$ & $1609(57 \%)$ & $3142(86 \%)$ & $<.0001$ \\
\hline Computed tomographic imaging & $2733(97 \%)$ & $3578(98 \%)$ & \\
\hline Pulmonary function tests & $1913(68 \%)$ & $3199(88 \%)$ & \\
\hline Preoperative mediastinal staging & $1621(57 \%)$ & $3142(86 \%)$ & \\
\hline $\begin{array}{l}\text { Intraoperative mediastinal } \\
\text { staging }\end{array}$ & $1609(57 \%)$ & $3321(91 \%)$ & \\
\hline $\begin{array}{l}\text { Preoperative chemotherapy or } \\
\text { radiation therapy }\end{array}$ & $26(0.9 \%)$ & $467(13 \%)$ & $<.0001$ \\
\hline No malignancy found & $257(9 \%)$ & $133(4 \%)$ & $<.0001$ \\
\hline Hospital stay $(\mathrm{d}$, mean $\pm \mathrm{SD})$ & $9 \pm 11$ & $6 \pm 7$ & $<.0001$ \\
\hline Prolonged stay & $213(8 \%)$ & $186(5 \%)$ & $<.0001$ \\
\hline Readmissions & $163(6 \%)$ & $249(7 \%)$ & .09 \\
\hline Charges (\$US, mean) & $\$ 89,000$ & $\$ 78,000$ & $<.0001$ \\
\hline
\end{tabular}

Values are numbers and percentages of patients unless otherwise stated. $S D$, Standard deviation.

investigators have found that outcomes after specific surgical procedures are superior when performed by surgeons who have undergone an additional formal training period after general surgery residency relative to general surgeons who have not done so. Hollenbeak and colleagues ${ }^{2}$ reported that patients undergoing carotid endarterectomy by a vascular surgeon had significantly lower rates of stroke and

TABLE 4. Morbidities and operative mortality

\begin{tabular}{lccc}
\hline & $\begin{array}{c}\text { General } \\
\text { surgeons }\end{array}$ & $\begin{array}{c}\text { Thoracic } \\
\text { surgeons }\end{array}$ & $\begin{array}{c}\boldsymbol{P} \\
\text { value }\end{array}$ \\
\hline Morbidities & $310(11 \%)$ & $146(4 \%)$ & $<.0001$ \\
Prolonged air leak $(>7 \mathrm{~d})$ & 136 & 42 & $<.0001$ \\
Reoperation for bleeding & 81 & 21 & $<.0001$ \\
Pneumonia & 32 & 25 & .07 \\
Myocardial infarction & 16 & 17 & .7 \\
Bronchopleural fistula & 8 & 4 & .19 \\
Respiratory failure & 29 & 23 & .1 \\
Stroke & 3 & 3 & .9 \\
Deep vein thrombosis & 5 & 11 & .5 \\
Mortality & $198(7 \%)$ & $73(2 \%)$ & $<.0001$ \\
Myocardial infarction & 74 & 29 & \\
Respiratory failure & 88 & 21 & \\
Multisystem organ failure or sepsis & 24 & 11 & \\
Stroke & 12 & 5 & \\
Pulmonary embolus & 9 & 7 & \\
\hline Data represent numbers of patients with percentages for overall categories.
\end{tabular}


reocclusion, as well as a shorter mean hospital stay than patients whose surgery was performed by a general surgeon. Similarly, Dimick and associates ${ }^{3}$ found significantly lower operative mortality after esophagectomy for thoracic surgeons relative to general surgeons.

Similar comparisons between cardiothoracic surgeons and general surgeons for patients undergoing lung cancer resection have also been published. Silvestri and colleagues ${ }^{4}$ from the Medical University of South Carolina published what is recognized as the initial modern comparison of the outcomes of lung cancer resection between general and cardiothoracic surgeons in 1998. Silvestri and colleagues ${ }^{4}$ found a significant reduction in operative mortality for patients undergoing either lobectomy or pneumonectomy performed by a cardiothoracic surgeon rather than general surgeons across the state of South Carolina; however, the difference in mortality may have been partially confounded by the inclusion of pneumonectomies in the analysis. Furthermore, no attempt to assess tumor staging was performed, and the study was geographically isolated. ${ }^{12}$

Goodney and coworkers ${ }^{5}$ subsequently published an analysis of Medicare claims data for the calendar year of 1998 , comparing the outcomes of lobectomy or pneumonectomy for lung cancer performed by either a general surgeon, a cardiac thoracic surgeon, or a noncardiac thoracic surgeon. They found significant differences in operative mortality between cardiac or noncardiac thoracic surgeons and general surgeons, differences that were in part related to hospital and surgeon volume. These findings, however, are tempered by the arbitrary and possibly inaccurate classification of cardiac versus noncardiac thoracic surgeons, a lack of inclusion of the stage of malignancy in the analysis, and the use of a single calendar year sample.

Schipper and colleagues ${ }^{1}$ and Ellis and associates ${ }^{6}$ have published the results of 2 retrospective cohort analyses comparing general surgeons, cardiac surgeons and noncardiac thoracic surgeons performing lung resections for lung cancer. In the 2009 article, ${ }^{1}$ a significant decrease in the frequency of operative mortality and prolonged stay was found for cardiac and noncardiac thoracic surgeons relative to general surgeons; when adjusted for surgeon volume, however, the difference in operative mortality dissipated.

In the 2011 report, $^{6}$ the Nationwide Inpatient Sample database was once more queried for lung resections for lung cancer. Again, a significant difference in operative mortality was found between cardiac and noncardiac thoracic surgeons as they were defined in the study relative to general surgeons (odds ratio, 1.47; $P=.003$ ). The investigators also found that preoperative and intraoperative surgical staging was significantly more frequent for noncardiac thoracic surgeons than for either general surgeons or cardiac thoracic surgeons $(73 \%, 55 \%$, and $54 \%$, respectively; $P<.0001$ ), which they hypothesized could explain superior perioperative and long-term outcomes. Although this study contributed valuable information to this topic, the assignment of surgeons to specialties without regard for board certification, a surrogate for a formal training period in thoracic surgery, may have confounded the results. Furthermore, the distribution of operative staging may explain long-term oncologic outcomes, as hypothesized by Farjah and associates, ${ }^{13}$ but it likely does not explain differences in operative morbidity and mortality.

This investigation sought to analyze the outcomes and patterns of care of patients undergoing lobectomy for NSCLC at multiple institutions across a geographically diverse health system. The use of the Premier inpatient database allowed all lobectomy procedures within the health care system, regardless of payer type, to be identified. This database also allowed patient charges, which have not been included in previous analyses, to be compared between the 2 groups. The addition of tumor registry data provided validation of some aspects of the Premiere data, as well as allowing the assessment of adherence to national treatment guidelines in the preoperative evaluation, a factor that we have previously found to improve the quality of care of patients with NSCLC. ${ }^{14}$

This study was also designed to limit operative procedures to lobectomy to minimize differences that could be expected with lesser procedures, such as nonanatomic wedge resections, and procedures with higher recognized rates of morbidity and mortality, such as pneumonectomy. Video-assisted thoracoscopic lobectomy was also excluded from this analysis, because the frequency of this procedure performed by general surgeons in this investigation was extremely low. Similarly, by limiting inclusion to $I C D-9$ codes that should allow resectability without the need for a complex lobectomy, it was anticipated that more uniform cohorts could be generated for analysis.

The classification of surgeons in this investigation was not based on their historical case mix but on their American Board of Medical Specialty board certification status. Board certification in either general or thoracic surgery should imply a requisite formal training period as well as some efforts to remain active and current in the specialty through the maintenance of certification process. Similarly, we chose not to separate board-certified thoracic surgeons into cardiac and noncardiac thoracic surgeons. According to the Thoracic Surgery Workforce Report published in $2011,{ }^{15}$ the majority of currently practicing thoracic surgeons who are not exclusively practicing congenital cardiac surgery in the United States continue to perform pulmonary resections, making an extrapolation of any meaningful results of this analysis to operational or health policy, difficult. Furthermore, although thoracic surgeons can limit their practice to adult cardiac or general thoracic surgery, there is no external validation of such a designation. 
On the basis of these study design parameters, an analysis of nearly 6500 lobectomies for NSCLC found that patients operated on by a thoracic surgeon were more likely to undergo a preoperative evaluation consistent with national guidelines and to have a reduced risk of operative morbidity relative to lobectomies performed by a general surgeons. Stay and mean hospital charges were also significantly reduced in the thoracic surgeon cohort. This is despite the findings that the thoracic surgeon patient cohort had a higher mean age, a higher rate of comorbid disease, more use of neoadjuvant therapy, and more advanced pathologic stage.

A significantly lower rate of operative mortality was also recognized in the thoracic surgeon patient cohort relative to the general surgeon patient cohort. This difference persisted even after adjustment of the analysis for facility or provider lobectomy volume. This is in contrast to some previous reports, in which adjustments for surgical volume negated the difference in mortality between similar groups. Possible explanations for these results include the assignment of surgeon specialty on the basis of formal training and board certification, the effect of separating cardiac and noncardiac thoracic surgeons in other reports, and a lower volume threshold for facility inclusion used in this review.

In light of the longer mean stay and the higher rate of postoperative morbidities, it is not surprising that the general surgeon patient cohort was found to have a higher mean hospital charge than that in the thoracic surgeon cohort. Seventy-six percent of the difference in mean charges can attributed to differences in stay and operative morbidity and mortality. The remaining difference in mean charges cannot be further defined and is probably related to individual practitioner variation. The difference in mean charges was statistically significant and, at a time when hospital margins of profit commonly range between $3 \%$ and $5 \%$, it is also operationally relevant.

Two other findings in this review raise concern regarding the adequacy of the preoperative evaluation some general surgeon patients underwent. Specifically, these include a higher rate of general surgeon cohort patients having surgery for what proved to be stage IIIB NSCLC and the higher frequency in the general surgeon patient cohort of surgical specimens in which no malignancy could be identified. Both of these findings may relate to the relatively low rates of computed tomographic and positron emission tomographic imaging use found in the general surgeon patient cohort.

This investigation has limitations common to similar analyses. Specifically, retrospective data are not as accurate as prospectively collected information. The best source of data for lobectomy for NSCLC, the Society of Thoracic Surgeons general thoracic surgery database, does not, however, include a significant number of surgical procedures performed by general surgeons to allow such a comparison. Some also do not consider the database to be reflective of the majority of cardiothoracic surgeons' practices at present because of low rates of participation.

Another potential criticism is that the outcome of a surgical procedure such as a lobectomy is the result of both the ability of the surgeon and the clinical processes and personnel inherent to a facility. Although adjusting the analysis for facility volume can partially compensate for this effect, qualitative differences may exist between facilities that do influence patient care and outcomes as previously shown. ${ }^{16}$ As Kozower and colleagues ${ }^{17}$ pointed out, such corrections are not always appropriate. Similarly, the effect of increasing surgical volume may not be beneficial above a specific threshold as seen in the work of Finley and associates ${ }^{18}$ in their analysis of Canadian hospitals performing lobectomy. Importantly, Treasure and colleagues ${ }^{19}$ found that within the specialty of cardiothoracic surgery volume does not significantly influence mortality after lobectomy, implying that differences found in outcomes between specialties in this investigation may be independent of volume and related to the additional training required for board certification in thoracic surgery. Finally, although they are theoretically pleasing, such adjustments do not change the fact that whatever the cause, patients in the general surgeon cohort face increased risks of both complications and death when undergoing lobectomy in the setting analyzed.

\section{CONCLUSIONS}

This review found that currently measurable indicators for quality of care were significantly superior and overall charges were significantly reduced when a lobectomy for NSCLC was performed by a cardiothoracic surgeon rather than by a general surgeon. These finding may have implications for facility and payer physician credentialing.

\section{References}

1. Schipper PH, Diggs BS, Ungerleider RM, Welke KF. The influence of surgeon specialty on outcomes in general thoracic surgery: a national sample 1996 to 2005. Ann Thorax Surg. 2009;88:1566-73.

2. Hollenbeak CS, Bowman AR, Harbaugh RE, Casale PN, Han D. The impact of surgical specialty on outcomes for carotid endarterectomy. J Surg Res. 2010;159: 595-602.

3. Dimick JB, Goodney PP, Orringer MB, Birkmeyer JD. Specialty training and mortality after esophageal cancer resection. Ann Thorac Surg. 2005;80:282-6.

4. Silvestri GA, Handy J, Lackland D, Corley E, Reed CE. Specialists achieve better outcomes than generalists for lung cancer surgery. Chest. 1998;114:675-80.

5. Goodney PP, Lucas FL, Stukel TA, Birkmeyer JD. Surgeon specialty and operative mortality with lung resection. Ann Surg. 2005;241:179-84.

6. Ellis MC, Diggs BS, Vetto JT, Schipper PH. Intraoperative oncologic staging and outcomes for lung cancer resection vary by surgeon specialty. Ann Thorac Surg. 2011;92:1958-64.

7. National Comprehensive Cancer Network Non-Small Cell Lung Cancer Panel. NCCN clinical practice guidelines in oncology (NCCN guidelines): non-small cell lung cancer. Version 3.2012; 2008. Fort Washington, PA: National Comprehensive Cancer Network. Available from: http://www.nccn.org/professionals/ physician_gls/pdf/nscl.pdf.

8. Iezzoni LI. Risk adjustment for measuring health care outcomes. 3rd ed. Chicago: Health Administration Press; 2003.

9. Quan H, Sundararajan V, Halfon P, Fong A, Burnand B, Luthi JC, et al. Coding algorithms for defining comorbidities in ICD-9-CM and ICD-10 administrative data. Med Care. 2005;43:1130-9. 
10. Cleverley WO, Song PH, Cleverley JO. Essentials of health care finance. Sudbury, MA: Jones \& Bartlett Learning; 2010;149-53.

11. Kozower BD, Sheng S, O'Brien SM, Liptay MJ, Lau CL, Jones DR, et al. STS database risk models: predictors of mortality and major morbidity for lung cancer resection. Ann Thorac Surg. 2010;90:875-83.

12. Kohman LJ. What constitutes success in cancer surgery? Measuring the value of specialist care. Chest. 1998;114:663-4.

13. Farjah F, Flum DR, Varghese TK Jr, Symons RG, Wood DE. Surgeon specialty and long-term survival after pulmonary resection for lung cancer. Ann Thorac Surg. 2009;87:995-1006.

14. Freeman RK, Van Woerkom J, Vyverberg A, Ascioti AJ. The effect of a multidisciplinary thoracic malignancy conference on the treatment of patients with lung cancer. Eur J Cardiothorac Surg. 2010;38:1-5.

15. Shemin RJ, Ikonomidis JS. Thoracic surgery workforce: report of STS/AATS Thoracic Surgery Practice and Access Task Force-snapshot 2010. J Thorac Cardiovasc Surg. 2012;143:39-46.

16. Billingsley KG, Morris AM, Dominitz JA, Matthews B, Dobie S, Barlow W, et al. Surgeon and hospital characteristics as predictors of major adverse outcomes following colon cancer surgery: understanding the volume-outcome relationship. Arch Surg. 2007;142:23-31

17. Kozower BD, Stukenborg GJ, Lau CL, Jones DR. Measuring the quality of surgical outcomes in general thoracic surgery: should surgical volume be used to direct patient referrals? Ann Thorac Surg. 2008;86:1405-8.

18. Finley CJ, Bendzsak A, Tomlinson G, Keshavjee S, Urbach DR, Darling GE. The effect of regionalization on outcome in pulmonary lobectomy: a Canadian national study. J Thorac Cardiovasc Surg. 2010;140:757-63.

19. Treasure T, Utley M, Bailey A. Assessment of whether in-hospital mortality for lobectomy is a useful standard for the quality of lung cancer surgery: retrospective study. BMJ. 2003;327:73

\section{Discussion}

Dr Thomas A. D'Amico (Durham, NC). Dr Freeman, I congratulate you and your colleagues on performing this important study. Improving the quality of care in the setting of the treatment of lung cancer should be the most important goal of future systems of care for cancer, because lung cancer is responsible for more than a third of all cancer deaths. Adherence to established guidelines, such as the NCCN guidelines, is the most efficient pathway to the improvement of outcomes. These guidelines in fact specify that patients with lung cancer should be treated by lung cancer specialists; that is, thoracic surgeons. Sadly, that recommendation has not reached the standard of care status in the United States.

This study, an analysis of more than 6400 patients in a single health care system, provides more evidence to support the NCCN's recommendations. Patients operated on by thoracic surgeons had significantly lower morbidity and mortality and also shorter stay despite being older and having a higher Charlson comorbidity index than patients operated on by general surgeons. As well, patients whose disease was staged and managed by thoracic surgeons received better evidence-based care, including preoperative and intraoperative mediastinal staging, all at a lower cost. Although there are acknowledged limitations of an administrative database analysis, as you outlined, the findings are persuasive; however, we are not the ones needing persuasion.

Although we would all agree that thoracic surgeons provide superior lung cancer care, does your research indicate that there were in fact thoracic surgeons who were not providing quality evidencebased care in this particular health care system? We will not be successful in changing paradigms of care if we are seen to be protecting inferior thoracic surgeons. Analysis of outcomes in the general thoracic surgery Society of Thoracic Surgeons database suggests that quality of lung cancer care is not universal in that database.

Dr Freeman. We were not, by agreement, able to focus on individual provider results with the health system. So I cannot tell you Dr X's mortality versus Dr Y's, and that was by design.

Dr D'Amico. So you will be seen as protecting inferior thoracic surgeons. The NCCN guidelines chosen for this study included the use of computed tomographic scanning and pulmonary function testing, relatively low thresholds for quality and nearly universally used by general and thoracic surgeons alike. Are there other parameters that you could have used or would use in the future to differentiate adherence to evidence-based guidelines more discriminatingly?

Dr Freeman. There certainly are other factors we could use. As you know, the hardest part of a study like this is not what to include but what not to include, because it has to be manageable. We chose what we thought were fairly low bar measures, such as pulmonary function tests, computed tomographic scan, and staging the mediastinum. We gave credit for positron emission tomography and mediastinoscopy. So we set the bar pretty low intentionally, as the first step. There are much more complex measures that we could use, and that may be something to look at in the future.

Dr D'Amico. Can you name what these measures are? What would you study to actually differentiate quality from not quality in the future?

Dr Freeman. One of the things I think is important personally, which is almost impossible to measure, unfortunately, is multidisciplinary care and multidisciplinary care conference planning. We found that to be extremely effective; transparency, multiple heads put around the table. It's very hard to measure though.

Dr D'Amico. Finally, what processes do you recommend to ascertain that only qualified surgeons are credentialed to manage patients with lung cancer in the future?

Dr Freeman. Well, I'm the eternal pessimist, so I have concerns that we as a physician group will ever be able to do that. One of the reasons we included cost in this and one of the reasons that we looked at a single health system is because I think the health system eventually will say, "Here are some data. Show us that you did additional training to do this procedure or you shouldn't be doing it." So I think it will have to be an external locus of control.

Dr D'Amico. Good paper. Thank you.

Dr Tomasz Grodzki (Szczecin, Poland). I congratulate you. I also have a short comment. I come from a country where the hospital charge for a lobectomy is US $\$ 5000$, and mortality and morbidity are exactly the same. The answer is because we convinced general surgeons in Poland that it's so complicated and dangerous to perform lobectomy that they are doing nothing, and $99 \%$ of lobectomies in Poland are performed by certified thoracic surgeons. This is the direction this Society should promote. It's simply dangerous to refer patients to general surgeons for lobectomy. If we compare it with the previous lecture by David Jones's group that the number of lung cancer cases in teaching hospitals is going down, the Society should fight for protecting the patients first and providing the patients with thoracic surgeons second. I think that the government relations committee or any of the Society committees should promote these results, because it's simply beneficial for the patients. 
Dr Freeman. Obviously, I agree in principle with your comments. As Dr Krasna would probably tell you, there are a lot of dilemmas regarding that kind of an idea. With $85 \%$ of cancer care in the United States occurring outside a National Cancer Institute cancer center, outside a university system, and geographically I'm not sure how you would make that work, but I think in principle you have a valid point.

Dr Mark J. Krasna (Neptune, $N J$ ). Rich, I think it was a great study. What I enjoyed, kind of looking at this as the other side of the good paper we heard this morning from UVA, is that since it was all one health system, although you did blind some of the issues that Tommy and I would have liked to have seen, you really took a deep dive into the data. So I'll just ask you the dirty question, which is, if you were the vice president of quality assurance of your health system, why is it when you're looking at the health system that so many lobectomies are being done for cancer by non-board certified thoracic surgeons, when in your health system you actually have a built-in referral opportunity to actually regionalize, which most of the hospitals don't even have that opportunity across the states?

Dr Freeman. I think it's multifactorial. Access is an issue. Yes, there are 76 hospitals in our system. Some are in Idaho, and some are in Indiana, and some are in Wisconsin. When you look at noncardiac thoracic surgeons in 76 hospitals, there are really 4 practices that have dedicated general thoracic surgeons. The vast majority of this practice is done by cardiothoracic surgeons. One thing that I do want to continue to look at and do some subset analysis on is the size of the hospital and who does the lobectomy. It's intuitive, but it's a general surgeon in a small hospital. Often it's not by choice. Often, when I talk to some of these people, it's the administration who actually wants them to do that case. So it's very complex.

Dr David M. Follette (Sacramento, Calif). I've been on a number of credentialing committees. The problem is that when they credential general surgeons, they look at their training and then they compare their numbers to the numbers that the American Board of Thoracic Surgery requires. For example, a number of years ago, the Board required 20 lobectomies. If a general surgeon documented 20 lobectomies, the credentials committees were in a very difficult position to deny them privileges. So the question is, in your regional system, how do you think you could get around this?

Dr Freeman. And that may work in our favor now. As most of you know, the general surgical residency training has changed significantly since most of us were general surgeons. Where senior level residents did a lot of noncardiac thoracic surgery, a lot of that has gone away; the requirements are much lower, and I think most general surgeons now come out with much less experience than they did 20 years ago in noncardiac thoracic surgery, unless they are very interested, at least in my experience.

Dr Stephen D. Cassivi (Rochester, Minn). Richard, I congratulate you. I think this is going to be an oft-quoted paper. Clearly, you're preaching to the converted here, and this is a message that needs to go beyond this room.

The argument countering your findings will be that you're a general thoracic surgeon and you're going to be biased. I just wanted to emphasize that I think you have bent over backwards to be fair in the construction of your comparison. You have compared open lobectomy with open lobectomy, whereas, as we know that video-assisted thoracoscopic surgical lobectomies are growing in number and are much more likely within the population of general thoracic surgeons. So your length of stay, your comorbidities, and your cost would likely show an even at a greater disparity if you were to compare general thoracic surgeons with general surgeons. It's more of a comment than a question, but I think it's something that we all need to underline as we go forward with these new data. Thanks very much for your presentation.

Dr Freeman. I think that's an excellent point. 